\title{
THE EVENTUAL SHAPE OF BETTI TABLES OF POWERS OF IDEALS
}

\author{
Amir Bagheri, Marc Chardin AND Huy TÀi HÀ
}

\begin{abstract}
Let $G$ be an abelian group and $S$ be a $G$-graded a Noetherian algebra over a commutative ring $A \subseteq S_{0}$. Let $I_{1}, \ldots, I_{s}$ be $G$-homogeneous ideals in $S$, and let $M$ be a finitely generated $G$-graded $S$-module. We show that the shape of non-zero $G$-graded Betti numbers of $M I_{1}^{t_{1}} \cdots I_{s}^{t_{s}}$ exhibit an eventual linear behavior as the $t_{i}$ s get large.
\end{abstract}

\section{Introduction}

It is a celebrated result (cf. $[5,12,15]$ ) that if $I \subseteq S$ is a homogeneous ideal in a Noetherian standard $\mathbb{N}$-graded algebra and $M$ is a finitely generated $\mathbb{Z}$-graded $S$ module then the regularity $\operatorname{reg}\left(I^{t} M\right)$ is asymptotically a linear function for $t \gg 0$. This asymptotic linear function and the stabilization index have also been studied in $[1,4,7-9]$.

In the case $S$ is a polynomial ring over a field, a more precise result is proved in [5]: the maximal degree of an $i$ th syzygy of $I^{t} M$ is eventually a linear function of $t$. Our interest here is to understand the eventual behavior of the degrees of all the minimal generators of the $i$ th syzygy module. This is of particular interest when the grading is given by some finitely generated abelian group $G$ that is not $\mathbb{Z}$, as in this case the result for the regularity of powers do not have an evident analogue. One can in particular consider the Cox ring of a toric variety, graded by the divisor class group.

We shall actually show, in the $G$-graded setting, that the collection of non-zero $G$-graded Betti numbers of $M I_{1}^{t_{1}} \ldots I_{s}^{t_{s}}$ exhibits an asymptotic linear behavior as the $t_{i}$ s get large. Let us also point out that, even when an explicit minimal free resolution of these powers is known, for instance when $I$ is a complete intersection ideal, the degrees of $i$ th syzygies of $I^{t}$ do not exhibit trivially a linear behavior.

Throughout the paper, let $G$ be a finitely generated abelian group and let $S=$ $A\left[x_{1}, \ldots, x_{n}\right]$ be a $G$-graded algebra over a commutative ring $A \subseteq S_{0}$. Hence $A=$ $S /\left(x_{1}, \ldots, x_{n}\right)$ is a $G$-graded $S$-module concentrated in degree 0 .

Our work hinges on the relationship between multigraded Betti numbers and the graded pieces of $\operatorname{Tor}_{i}^{S}\left(M I_{1}^{t_{1}} \cdots I_{s}^{t_{s}}, A\right)$; we, thus, examine the support of the module $\operatorname{Tor}_{i}^{S}\left(M I_{1}^{t_{1}} \cdots I_{s}^{t_{s}}, A\right)$ as the $t_{i}$ s get large. Our main result, in the case of a single ideal, gives the following:

Theorem 1.1 (see Theorem 4.6). Let $I=\left(f_{1}, \ldots, f_{r}\right)$ be a G-homogeneous $S$-ideal, and let $M$ be a finitely generated $G$-graded $S$-module. Set $\Gamma:=\left\{\operatorname{deg}_{G}\left(f_{i}\right)\right\}_{i=1}^{r}$.

Received by the editors February 4, 2013.

2000 Mathematics Subject Classification. 13D45, 13D02.

Keywords and phrases. Betti numbers, asymptotic linearity, multigraded. 
Let $\ell \geq 0$, and assume that $\ell=0$ or $A$ is Noetherian.

There exist a finite collection of elements $\delta_{i} \in G$, a finite collection of integers $t_{i}$, and a finite collection of non-empty tuples $E_{i}=\left(\gamma_{i, 1}, \ldots, \gamma_{i, s_{i}}\right)$ of elements in $\Gamma$, such that the elements $\left(\gamma_{i, j+1}-\gamma_{i, j}, 1 \leq j \leq s_{i}-1\right)$ are linearly independent, satisfying

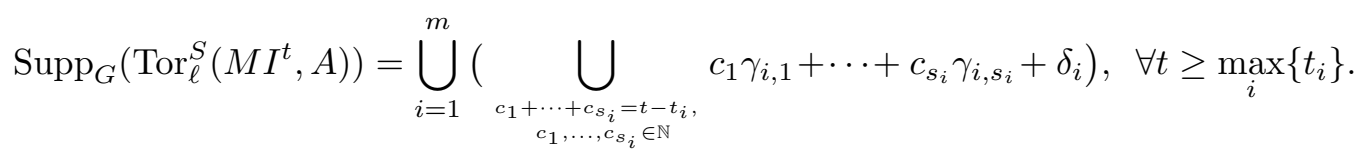

The condition of linear independence stated for $E_{i}$ implies that $c_{1} \gamma_{i, 1}+\cdots+$ $c_{s_{i}} \gamma_{i, s_{i}} \neq c_{1}^{\prime} \gamma_{i, 1}+\cdots+c_{s_{i}}^{\prime} \gamma_{i, s_{i}}$ if $c_{1}+\cdots+c_{s_{i}}=c_{1}^{\prime}+\cdots+c_{s_{i}}^{\prime}$ and $\left(c_{1}, \ldots, c_{s_{i}}\right) \neq$ $\left(c_{1}^{\prime}, \ldots, c_{s_{i}}^{\prime}\right)$. Note the important fact that the elements in $E_{i}$ are all in $\Gamma$, the set of degrees of generators of $I$.

Theorem 4.6 is proved in the last section of the paper. Our proof is based on the two following observations. Firstly, the multi-Rees module (sometimes also referred to as the Rees modification) $M \mathcal{R}=\bigoplus_{t_{i} \geq 0} M I_{1}^{t_{1}} \ldots I_{s}^{t_{s}}$ is a $G \times \mathbb{Z}^{s}$-graded module over the multi-Rees algebra $\mathcal{R}=\bigoplus_{t_{i}>0} I_{1}^{t_{1}} \ldots I_{s}^{t_{s}}$. Secondly, if $G^{\prime}$ is a finitely generated abelian group and $R=S\left[T_{1}, \ldots, T_{r}\right]$ is a $G \times G^{\prime}$-graded polynomial extension of $S$, such that $\operatorname{deg}_{G \times G^{\prime}}(a)=\left(\operatorname{deg}_{G}(a), 0\right)$ for any $a \in S$, then for a graded complex $\mathbb{G}_{\bullet}$ of free $R$-modules,

$$
H_{i}\left(\left(\mathbb{G}_{\bullet}\right)_{G \times\{\delta\}} \otimes_{S} A\right)=H_{i}\left(\mathbb{G} \bullet \otimes_{S} A\right)_{G \times\{\delta\}},
$$

where $(\bullet)_{G \times\{\delta\}}$ denotes the degree $G \times\{\delta\}$-strand of the corresponding complex. In particular, if $\mathbb{G}_{\bullet}$ is a $G \times G^{\prime}$-graded free resolution of an $R$-module $N$, as $\left(\mathbb{G}_{\bullet}\right)_{G \times\{\delta\}}$ is a $G$-graded free resolution of the $S$-module $N_{G \times\{\delta\}}$, it follows that

$$
\operatorname{Tor}_{i}^{S}\left(N_{G \times\{\delta\}}, A\right)=H_{i}\left(\mathbb{G}_{\bullet} \otimes_{S} A\right)_{G \times\{\delta\}},
$$

in which $\mathbb{G} \bullet \otimes_{S} A$ is viewed as a $G \times G^{\prime}$-graded complex of free $A\left[T_{1}, \ldots, T_{r}\right]$-modules. These observations allow us to bring the problem to studying the support of graded $A\left[T_{1}, \ldots, T_{r}\right]$-modules. We proceed by making use of the notion of initial submodules to reduce to the case when the module is a quotient ring obtained by a monomial ideal. The result then follows by considering the Stanley decomposition of such a quotient ring.

In its full generality, our proof of Theorem 4.6 is quite technical, so we start in Section 3 by considering first the case when each $I_{i}$ is equi-generated. In fact, in this case, with the additional assumption that $A$ is a Noetherian ring, our results are much stronger; the asymptotic linearity appears clearer and the proofs are simpler. We can also examine the support of local cohomology modules of $M I_{1}^{t_{1}} \cdots I_{s}^{t_{s}}$. Our results, in the case when each $I_{i}$ is finitely generated in a single degree $\gamma_{i}$, are stated as follows.

Theorem 1.2 (Theorem 3.3). Assume that $i=0$ or $A$ is Noetherian. Then there exists a finite set $\Delta_{i} \subseteq G$ such that

(1) For all $=\left(t_{1}, \ldots, t_{s}\right) \in \mathbb{N}^{s}$, $\operatorname{Tor}_{i}^{S}\left(M I_{1}^{t_{1}} \cdots I_{s}^{t_{s}}, A\right)_{\eta}=0$ if $\eta \notin \Delta_{i}+t_{1} \gamma_{1}+\cdots+t_{s} \gamma_{s}$.

(2) There exists a subset $\Delta_{i}^{\prime} \subseteq \Delta_{i}$ such that $\operatorname{Tor}_{i}^{S}\left(M I_{1}^{t_{1}} \cdots I_{s}^{t_{s}}, A\right)_{\eta+t_{1} \gamma_{1}+\cdots+t_{s} \gamma_{s}} \neq 0$ for $t \gg 0$ and $\eta \in \Delta_{i}^{\prime}$, and $\operatorname{Tor}_{i}^{S}\left(M I_{1}^{t_{1}} \cdots I_{s}^{t_{s}}, A\right)_{\eta+t_{1} \gamma_{1}+\cdots+t_{s} \gamma_{s}}=0$ for $t \gg 0$ and $\eta \notin \Delta_{i}^{\prime}$. 
(3) Let $A \rightarrow k$ be a ring homomorphism to a field $k$. Then for any $\delta$ and any $j$, the function

$$
\operatorname{dim}_{k} \operatorname{Tor}_{j}^{A}\left(\operatorname{Tor}_{i}^{S}\left(M I_{1}^{t_{1}} \cdots I_{s}^{t_{s}}, A\right)_{\delta+t_{1} \gamma_{1}+\cdots+t_{s} \gamma_{s}}, k\right)
$$

is polynomial in the $t_{i} s$ for $t \gg 0$, and the function

$$
\operatorname{dim}_{k} \operatorname{Tor}_{i}^{S}\left(M I_{1}^{t_{1}} \cdots I_{s}^{t_{s}}, k\right)_{\delta+t_{1} \gamma_{1}+\cdots+t_{s} \gamma_{s}}
$$

is polynomial in the $t_{i} s$ for $t \gg 0$.

Theorem 1.3 (Theorem 3.4). Let $\mathfrak{b}$ be a G-homogeneous ideal in $S$ such that for any $i \geq 0$ and $\delta \in G, H_{\mathfrak{b}}^{i}(S)_{\delta}$ is a finitely generated $A$-module. Then, if $A$ is Noetherian, for $i \geq 0$, there exists a subset $\Lambda_{i} \subseteq G$ such that

(1) $H_{\mathfrak{b}}^{i}\left(M I_{1}^{t_{1}} \cdots I_{s}^{t_{s}}\right)_{\eta+t_{1} \gamma_{1}+\cdots+t_{s} \gamma_{s}} \neq 0$ for $t=\left(t_{1}, \ldots, t_{s}\right) \gg 0$ if only if $\eta \in \Lambda_{i}$.

(2) Let $A \rightarrow k$ be a ring homomorphism to a field $k$. Then for any $\delta$ and any $j$, the function

$$
\operatorname{dim}_{k} \operatorname{Tor}_{j}^{A}\left(H_{\mathfrak{b}}^{i}\left(M I_{1}^{t_{1}} \cdots I_{s}^{t_{s}}\right)_{\delta+t_{1} \gamma_{1}+\cdots+t_{s} \gamma_{s}}, k\right)
$$

is a polynomial in the $t_{i} s$ for $t \gg 0$.

In the simplest scenario, when $S$ is a standard graded polynomial ring over a field $k, \mathfrak{m} \subseteq S$ the homogeneous maximal $S$-ideal, $s=1$, and $I \subseteq S$ is a homogeneous ideal generated in degree $d$, Theorems 1.2 and 1.3 give the following result.

Corollary 1.4. For $i \geq 0$, there exist $t_{i}$ and finite sets $\Delta_{i}^{\prime} \subseteq \Delta_{i} \subseteq \mathbb{Z}$ such that

(1) for all $t \in \mathbb{N}$, $\operatorname{Tor}_{i}^{S}\left(M I^{t}, k\right)_{\eta+t d}=0$ if $\eta \notin \Delta_{i}$;

(2) for $t \geq t_{i}, \operatorname{Tor}_{i}^{S}\left(M I^{t}, k\right)_{\eta+t d} \neq 0$ if and only if $\eta \in \Delta_{i}^{\prime}$;

(3) for any $\eta \in \mathbb{Z}$, the function $\operatorname{dim}_{k} \operatorname{Tor}_{i}^{S}\left(M I^{t}, k\right)_{\eta+t d}$ is a polynomial in $t$ for $t \geq t_{i}$;

(4) for any $\theta \in \mathbb{Z}$, the function $\operatorname{dim}_{k} H_{\mathfrak{m}}^{i}\left(M I^{t}\right)_{\theta+t d}$ is a polynomial in $t$, for $t \gg 0$.

While writing this paper, we were informed by Whieldon that in her recent work [16], a similar result to Corollary $1.4(1)-(3)$ is proved. We later learned that Pooja Singla also proved these results, in the second chapter of her thesis [14], independently. She also shows in [14] that if $I$ is graded ideal, then for any $a, b \in \mathbb{Z}$, $\operatorname{dim}_{k} \operatorname{Tor}_{i}^{S}\left(I^{t}, k\right)_{a+b t}$ is a quasi-polynomial in $t$ for $t \gg 0$, and give results describing the regularity of $I_{1}^{t_{1}} \cdots I_{s}^{t_{s}}$ for $t \gg 0$.

In the general setting, when $A$ is not necessarily a field and $S$ is not necessarily standard graded, the problem is more subtle, and requires more work and a different approach.

We choose not to restrict to a Noetherian base ring in all our results as it seemed to us that it makes the presentation more clear to put this hypothesis only in the statements where it is of use in the proof.

\section{Preliminaries}

In this section, we collect necessary notations and terminology used in the paper, and prove a few auxiliary results. For basic facts in commutative algebra, we refer the reader to $[6,13]$. 
From now on, $G$ denotes a finitely generated abelian group, $S=A\left[x_{1}, \ldots, x_{n}\right]$ is a $G$-graded algebra over a commutative ring with identity, $A \subseteq S_{0}$ and $M$ is a $G$ graded $S$-module. By abusing notation, we shall use 0 to denote the identity of all abelian groups considered in the paper; the particular group will be understood from the context of its use.

Definition 2.1. Let $E \subseteq G$ be a collection of elements in $G$. We say that $E$ is a linearly independent subset of $G$ if $E$ forms a basis for a free submonoid of $G$.

Definition 2.2. The support of $M$ in $G$ is defined to be

$$
\operatorname{Supp}_{G}(M)=\left\{\gamma \in G \mid M_{\gamma} \neq 0\right\} .
$$

Remark 2.3. When $A$ is a field, let $\mathbb{F}$. be a minimal $G$-graded free resolution of $M$ over $S$, where

$$
\mathbb{F}_{i}=\bigoplus_{\eta \in G} S(-\eta)^{\beta_{\eta}^{i}(M)}
$$

The numbers $\beta_{\eta}^{i}(M)$ are called the multigraded (or G-graded) Betti numbers of $M$ and

$$
\beta_{\eta}^{i}(M)=\operatorname{dim}_{A} \operatorname{Tor}_{i}^{S}(M, A)_{\eta}
$$

as, by definition, the maps in $\mathbb{F} \bullet \otimes_{S} A$ are zero maps.

More generally, we shall prove the following lemma relating the multigraded Betti numbers of $M$ and the support of $\operatorname{Tor}_{*}^{S}(M, A)$.

Lemma 2.4. Let $\mathbb{F} \bullet$ be a $G$-graded free resolution of a $G$-graded $S$-module $M$. Then (1) $\mathbb{F}_{i}$ has a summand $S(-\gamma)$ for any $\gamma \in \operatorname{Supp}_{G}\left(\operatorname{Tor}_{i}^{S}(M, A)\right)$.

(2) Assume that there exists $\phi \in \operatorname{Hom}_{\mathbb{Z}}(G, \mathbb{R})$ such that $\phi\left(\operatorname{deg}\left(x_{i}\right)\right)>0$ for all $i$ and $M$ is finitely generated. Then there exists a G-graded free resolution $\mathbb{F}^{\prime}$ of $M$ such that

$$
\mathbb{F}_{i}^{\prime}=\bigoplus_{\ell \in E_{i}^{\prime}} S\left(-\gamma_{i, \ell}\right) \quad \text { with } \quad \gamma_{i, \ell} \in \bigcup_{j \leq i} \operatorname{Supp}_{G}\left(\operatorname{Tor}_{j}^{S}(M, A)\right), \forall \ell
$$

Note that, without further restrictions on $A$ and/or $M$ one cannot in general choose $\mathbb{F}_{i}^{\prime}$ so that $\gamma_{i, \ell} \in \operatorname{Supp}_{G}\left(\operatorname{Tor}_{i}^{S}(M, A)\right), \forall \ell$.

Proof. For (1), let $K$ be defined by the exact sequence

$$
0 \rightarrow K \rightarrow \mathbb{F}_{0} \rightarrow M \rightarrow 0
$$

and note that $\mathbb{F}_{0} \otimes_{S} A \rightarrow M \otimes_{S} A$ is onto. As $\left(\mathbb{F}_{0} \otimes_{S} A\right)_{\gamma} \neq 0$ if and only if $S(-\gamma)$ is a direct summand of $\mathbb{F}_{0}$, the result holds for $i=0$. Furthermore, $\cdots \rightarrow \mathbb{F}_{1} \rightarrow K \rightarrow 0$ is a resolution of $K, \operatorname{Tor}_{1}^{S}(M, A)$ is a graded submodule of $K \otimes_{S} A$ and $\operatorname{Tor}_{i}^{S}(M, A) \simeq$ $\operatorname{Tor}_{i-1}^{S}(K, A)$ for $i \geq 2$, which implies the result by induction on $i$.

To prove the second statement, we relax the finite generation of $M$ by the following condition, which will enable us to make induction : $\exists q \in \mathbb{R}, \phi(\operatorname{deg} a)>q, \forall a \in M$. Note that if a module satisfies this condition, any of its submodules satisfies the same condition. 
Set $T_{j}:=\operatorname{Supp}_{G}\left(\operatorname{Tor}_{j}^{S}(M, A)\right)$. Let $\psi: \mathbb{F}_{0}=\bigoplus_{\ell \in E_{0}} S\left(-\gamma_{0, \ell}\right) \rightarrow M$ be the augmentation and $E_{0}^{\prime}:=\left\{\ell \in E_{0} \mid \gamma_{0, \ell} \in T_{0}\right\}$. Denote by $\psi^{\prime}$ the restriction of $\psi$ to $\mathbb{F}_{0}^{\prime}:=\bigoplus_{\ell \in E_{0}^{\prime}} S\left(-\gamma_{0, \ell}\right)$.

We now prove that $\psi^{\prime}$ is onto. First, note that $\psi^{\prime} \otimes_{S} A$ is surjective. Assume that $\psi^{\prime}$ is not surjective, let $M^{\prime}$ be the image of $\psi^{\prime}$ and

$$
h:=\inf \left\{\phi(\gamma), M_{\gamma} \neq M_{\gamma}^{\prime}\right\} \geq \inf \{\phi(\operatorname{deg}(a)), a \in M\} \in \mathbb{R} .
$$

Set $\epsilon:=\min \left\{\phi\left(\operatorname{deg}\left(x_{i}\right)\right), i=1, \ldots, n\right\}>0$ and choose $m \in M_{\nu} \backslash M_{\nu}^{\prime}$ for some $\nu$ with $h \leq \phi(\nu)<h+\epsilon$. As $\psi^{\prime} \otimes_{S} A$ is onto, there exists $m^{\prime} \in M^{\prime}$ such that $m$ is of the form $m^{\prime}+\sum_{i=1}^{n} m_{i} x_{i}$. Now, for some value $i$ we have $m_{i} \notin M^{\prime}$. It then follows that $\phi\left(\operatorname{deg}\left(m_{i}\right)\right)=\phi(\nu)-\phi\left(\operatorname{deg}\left(x_{i}\right)\right)<h$, contradicting the definition of $h$.

We will now prove (2) by induction on $i$. To end this, assume that there exists a graded complex

$$
0 \rightarrow \mathbb{F}_{i}^{\prime} \rightarrow \cdots \rightarrow \mathbb{F}_{0}^{\prime} \rightarrow M \rightarrow 0
$$

with at most non-zero homology in homological degree $i \geq 0$, and such that $\mathbb{F}_{i}^{\prime}=$ $\bigoplus_{\ell \in E_{i}^{\prime}} S\left(-\gamma_{i, \ell}\right)$ with $\gamma_{i, \ell} \in \bigcup_{j \leq i} T_{j}$.

If the complex is exact, our claim is proved; otherwise, by setting $K_{i} \subset \mathbb{F}_{i}^{\prime}$ for the $i$ th homology module of the complex and $Q_{i}:=\operatorname{ker}\left(\mathbb{F}_{i}^{\prime} \otimes_{S} A \rightarrow \mathbb{F}_{i-1}^{\prime} \otimes_{S} A\right)$, one has

$$
\operatorname{Supp}_{G}\left(K_{i} \otimes_{S} A\right)=\operatorname{Supp}_{G}\left(\operatorname{Tor}_{i+1}^{S}(M, A)\right) \cup \operatorname{Supp}_{G}\left(\operatorname{ker}\left(Q_{i} \rightarrow \operatorname{Tor}_{i}^{S}(M, A)\right)\right) .
$$

Applying the argument above to a graded onto map $\mathbb{F} \rightarrow K_{i}$, and using that by induction

$$
\operatorname{Supp}_{G}\left(K_{i} \otimes_{S} A\right) \subseteq T_{i+1} \cup \operatorname{Supp}_{G}\left(\mathbb{F}_{i}^{\prime} \otimes_{S} A\right) \subseteq \cup_{j \leq i+1} T_{j}
$$

one obtains a graded free $S$-module $\mathbb{F}_{i+1}^{\prime}$ as claimed mapping onto $K_{i}$.

Let $t=\left(t_{1}, \ldots, t_{s}\right) \in \mathbb{Z}^{s}$. We shall write $t \geq 0$ (respectively, $t>0, t \leq 0$ and $t \neq 0$ ) if $t_{i} \geq 0$ (respectively, $t_{i}>0, t_{i} \leq 0$ and $t_{i} \neq 0$ ) for all $i=1, \ldots, s$. For a property that depends on a parameter $t \in \mathbb{Z}^{s}$, one says that the property holds for $t \gg 0$ if there exists $t_{0} \in \mathbb{Z}^{s}$ such that it holds for $t \in t_{0}+\mathbb{N}^{s}$. The following semi-classical lemma will be of use.

Lemma 2.5. Let $R$ be a finitely generated $\mathbb{N}^{s}$-graded algebra over a commutative ring $A$. Let $M$ be a finitely generated $\mathbb{Z}^{s}$-graded $R$-module. Then either $M_{t}=0$ for $t \gg 0$ or $M_{t} \neq 0$ for $t \gg 0$.

Proof. Let $\mathfrak{b}=\bigoplus_{t_{i} \geq 1} R_{\left(t_{1}, \ldots, t_{s}\right)}$ be the ideal generated by elements of strictly positive degrees. If $M \neq H_{\mathfrak{b}}^{0}(M)$ one has $M_{t} \neq 0$ for any $t \in t_{0}+\mathbb{N}^{s}$, where $t_{0}$ is the degree of a non-zero element in $M / H_{\mathfrak{b}}^{0}(M)$.

If $M=H_{\mathfrak{b}}^{0}(M)$ then any generator $a$ of $M$ spans a submodule of $M$ that is zero in degrees $\operatorname{deg}(a)+b_{a}(1, \ldots, 1)+\mathbb{N}^{s}$, where $b_{a}$ is such that for any product $p$ of $b_{a}$ elements among the finitely many generators of the $R$-ideal $\mathfrak{b}$, one has $p a=0$. As $M$ is finitely generated, the result follows.

We shall make use of the notion of initial modules with respect to a monomial order. This is a natural extension of the familiar notion of initial ideals in a polynomial ring. Let $F$ be a free $S$-module. We can write $F=\bigoplus_{i \in I} S e_{i}$. A monomial in $F$ is of the 
form $\mathbf{x}^{\alpha} e_{i}$, where $\mathbf{x}^{\alpha}$ is a monomial in $S$ and $i \in I$. A monomial order in $F$ is a total order, say $\prec$, on the monomials of $F$ satisfying the following condition:

if $u \prec v$ and $w \neq 1$ is a monomial in $S$ then $u \prec u w \prec v w$.

It can be seen that $\prec$ is a well ordering, i.e., every non-empty subset of the monomials in $F$ has a minimal element. We refer the reader to $[6,15.2]$ for more details on monomial orders on free modules.

Definition 2.6. Let $F$ be a free $S$-module, and let $K$ be an $S$-submodule of $F$. Let $\prec$ be a monomial order in $F$. The initial module of $K$, denoted by $\operatorname{in}_{\prec}(K)$, is defined to be the $S$-submodule of $F$ generated by

$$
\left\{\mathbf{x}^{\alpha} e_{i} \mid \exists f=\mathbf{x}^{\alpha} e_{i}+\text { smaller terms } \in K\right\} .
$$

Proposition 2.7. Let $F$ be a free $G$-graded $S$-module, and let $K$ be a $G$-graded $S$-submodule of $F$. Let $\prec$ be a monomial order in $F$. Then $\operatorname{in}_{\prec}(K)$ is a $G$-graded $S$-module of $F$, and

$$
\operatorname{Supp}_{G}(F / K)=\operatorname{Supp}_{G}\left(F / \operatorname{in}_{\prec}(K)\right) .
$$

Proof. It is clear from the definition that $\operatorname{in}_{\prec}(K)$ is a $G$-graded $S$-module. To prove the proposition, we need to show that for any $\mu \in G, K_{\mu}=F_{\mu}$ if and only if $\operatorname{in}_{\prec}(K)_{\mu}=F_{\mu}$.

Clearly, if $K_{\mu}=F_{\mu}$ then all monomials of degree $\mu$ in $F$ are elements of $K$, and thus, are elements of $\operatorname{in}_{\prec}(K)$. Therefore, in this case, $\operatorname{in}_{\prec}(K)_{\mu}=F_{\mu}$.

Suppose now that in ${ }_{\prec}(K)_{\mu}=F_{\mu}$. Let $\mathbf{x}^{\alpha} e_{i}$ be the smallest monomial of degree $\mu$ in $F$ but not in $K$, if it exists. Then $\mathbf{x}^{\alpha} e_{i} \in \operatorname{in}_{\prec}(K)_{\mu}$. Thus, there exists an element $f \in K$ of the form

$$
f=\mathbf{x}^{\alpha} e_{i}+g,
$$

where $g$ consists of monomials that are smaller than $\mathbf{x}^{\alpha} e_{i}$ with respect to $\prec$. Since $K$ is a $G$-graded $S$-module, we can choose $f$ to be $G$-homogeneous of degree $\mu$. That is, all its monomials are of degree $\mu$. This implies that all monomials, and thus, all terms in $g$ are elements in $K$. In particular, $g \in K$. Therefore, $\mathbf{x}^{\alpha} e_{i}=f-g \in K$, a contradiction. Hence, $F_{\mu}=K_{\mu}$. The proposition is proved.

One of our techniques is to take the collection of elements of certain degrees from a complex. This construction gives what we shall call strands of the complex.

Definition 2.8. Let $\mathbb{F}$. be a $G$-graded complex of $S$-modules and let $\Gamma \subseteq G$. The $\Gamma$-strand of $\mathbb{F}_{\bullet}$, often denoted by $\left(\mathbb{F}_{\bullet}\right)_{\Gamma}$, is obtained by taking elements of degrees belonging to $\Gamma$ in $\mathbb{F} \bullet$ and the boundary maps between these elements (since the complex is graded, the boundary maps are of degree 0 ). In particular, if $F=\bigoplus_{\gamma \in G} F_{\gamma}$ is a $G$-graded $S$-module, then $F_{\Gamma}:=\bigoplus_{\gamma \in \Gamma} F_{\gamma}$. Note that the degree $\Gamma$-strand of a complex/module is not necessarily a complex/module over $S$.

\section{Forms of the same degree}

In this section, we consider the case when every ideal $I_{i}$ is generated in a single degree. That is, when $\operatorname{deg}_{G}\left(f_{i, j}\right)=\gamma_{i}$ for all $j$. We keep the notations of Section 2 . 
Let $G^{\prime}$ denote a finitely generated abelian group. The following result, with $G^{\prime}=$ $\mathbb{Z}^{s}$, will be a key ingredient of our proof.

Theorem 3.1. Let $R=S\left[T_{1}, \ldots, T_{r}\right]$ be a $G \times G^{\prime}$-graded polynomial extension of $S$ with $\operatorname{deg}_{G \times G^{\prime}}(a) \in G \times 0$ for all $a \in S$ and $\operatorname{deg}_{G \times G^{\prime}}\left(T_{j}\right) \in 0 \times G^{\prime}$ for all $j$. Let $\mathbb{M}$ be a finitely generated $G \times G^{\prime}$-graded $R$-module and let $i$ be an integer. Assume that $i=0$ or $A$ is a Noetherian ring. Then

(1) There exists a finite subset $\Delta_{i} \subseteq G$ such that, for any $t, \operatorname{Tor}_{i}^{S}\left(\mathbb{M}_{(*, t)}, A\right)_{\delta}=0$ for all $\delta \notin \Delta_{i}$.

(2) Assume that $G^{\prime}=\mathbb{Z}^{s}$. For $\delta \in \Delta_{i}$, $\operatorname{Tor}_{i}^{S}\left(\mathbb{M}_{(*, t)}, A\right)_{\delta}=0$ for $t \gg 0$ or $\operatorname{Tor}_{i}^{S}$ $\left(\mathbb{M}_{(*, t)}, A\right)_{\delta} \neq 0$ for $t \gg 0$. If, furthermore, $A \rightarrow k$ is a ring homomorphism to $a$ field $k$, then for any $j$, the function

$$
\operatorname{dim}_{k} \operatorname{Tor}_{j}^{A}\left(\operatorname{Tor}_{i}^{S}\left(\mathbb{M}_{(*, t)}, A\right)_{\delta}, k\right)
$$

is polynomial in the $t_{i} s$ for $t \gg 0$, and the function

$$
\operatorname{dim}_{k} \operatorname{Tor}_{i}^{S}\left(\mathbb{M}_{(*, t)}, k\right)_{\delta}
$$

is polynomial in the $t_{i} s$ for $t \gg 0$.

Proof. Let $\mathbb{F} \bullet$ be a graded free resolution of $\mathbb{M}$ over $R$, where $\mathbb{F}_{i}=\bigoplus_{\eta, j} R(-\eta,-j)^{\beta_{\eta, j}^{i}}$ is of finite rank for $i=0$, and for any $i$ when $A$ is Noetherian. For $t \in G^{\prime}$, the $(*, t)$ strand of $\mathbb{F}_{\bullet}$, denoted by $\mathbb{F}_{\bullet}^{t}$, is a $G$-graded free resolution of $\mathbb{M}_{(*, t)}$ over $S=R_{(*, 0)}$, that is not necessarily minimal. Its $i$ th term is

$$
\mathbb{F}_{i}^{t}=\bigoplus_{\eta, j} S(-\eta)^{\beta_{\eta, j}^{i}} \otimes_{A} B_{t-j}
$$

where $B=A\left[T_{1}, \ldots, T_{r}\right]$.

Let $\Delta_{i}=\left\{\eta \mid \exists j: \beta_{\eta, j}^{i} \neq 0\right\}$. The module $\operatorname{Tor}_{i}^{S}\left(\mathbb{M}_{(*, t)}, A\right)=H_{i}\left(\mathbb{F}_{\bullet}^{t} \otimes_{S} A\right)$ is a subquotient of the module $\bigoplus_{\eta, j} A(-\eta)^{\beta_{\eta, j}^{i}} \otimes_{A} B_{t-j}$, and (1) is proved.

To prove (2), observe first that $\operatorname{Tor}_{i}^{S}\left(\mathbb{M}_{(*, t)}, A\right)_{\delta}=H_{i}\left(\mathbb{F}_{\bullet}^{t} \otimes_{S} A\right)_{\delta}$ and $\left(A(-\eta) \otimes_{A}\right.$ $\left.B_{t-j}\right)_{\delta}=A_{\delta-\eta} \otimes_{A} B_{t-j}$ is zero if $\eta \neq \delta$. Thus, $H_{i}\left(\mathbb{F}_{\bullet}^{t} \otimes_{S} A\right)_{\delta}$ is equal to $H_{i}\left(\mathbb{F}_{\bullet}^{[\delta]} \otimes_{S} A\right)_{t}$, where $\mathbb{F}_{\bullet}^{[\delta]}$ is the subcomplex of $\mathbb{F} \bullet$ given by

$$
\mathbb{F}_{i}^{[\delta]}=\bigoplus_{j} R(-\delta,-j)^{\beta_{\delta, j}^{i}}=\bigoplus_{j}\left[S(-\delta) \otimes_{A} B(-j)\right]^{\beta_{\delta, j}^{i}} .
$$

As $\mathbb{F}_{\bullet}^{[\delta]} \otimes_{S} A$ is a graded complex of finitely generated $B$-modules, $H_{i}\left(\mathbb{F}_{\bullet}^{[\delta]} \otimes_{S} A\right)$ is a finitely generated $B$-module for any $i$ when $A$ is Noetherian. Similarly, $\operatorname{Tor}_{i}^{S}\left(\mathbb{M}_{(*, t)}, k\right)_{\delta}$ $=H_{i}\left(\mathbb{F}_{\bullet}^{t} \otimes_{S} k\right)_{\delta}=H_{i}\left(\mathbb{F}_{\bullet}^{[\delta]} \otimes_{S} k\right)$ is a finitely generated $k\left[T_{1}, \ldots, T_{r}\right]$-module for any $i$ when $A$ is Noetherian.

This proves (2) in view of Lemma 2.5 and [11, Theorem 1].

Remark 3.2. In the context of point (2) above, there is a graded spectral sequence of graded $B$-modules with second term $E_{j, i}^{2}(t)=\operatorname{Tor}_{j}^{A}\left(\operatorname{Tor}_{i}^{S}\left(\mathbb{M}_{(*, t)}, A\right)_{\delta}, k\right)$ that converges to $\operatorname{Tor}_{i+j}^{S}\left(\mathbb{M}_{(*, t)}, k\right)_{\delta}$. It follows that all terms $E_{j, i}^{p}(t)$ for $p \geq 2$ are finitely generated $k\left[T_{1}, \ldots, T_{r}\right]$-modules. In particular, one can write :

$$
\operatorname{dim}_{k} \operatorname{Tor}_{\ell}^{S}\left(\mathbb{M}_{(*, t)}, k\right)_{\delta}=\sum_{i+j=\ell} \operatorname{dim}_{k} E_{j, i}^{\infty}(t) \leq \sum_{i+j=\ell} \operatorname{dim}_{k} E_{j, i}^{p}(t), \quad \forall p \geq 2,
$$


which provides a control on the Hilbert function (and polynomial) of $\operatorname{Tor}_{\ell}^{S}(\mathbb{M}, k)_{\delta}$ in terms of the ones of $\operatorname{Tor}_{j}^{A}\left(\operatorname{Tor}_{i}^{S}(\mathbb{M}, A)_{\delta}, k\right)$ for $i+j=\ell$.

We are now ready to examine the asymptotic linear behavior of non-zero $G$-graded Betti numbers and non-vanishing degrees of local cohomology modules of $M I_{1}^{t_{1}} \cdots I_{s}^{t_{s}}$.

In the next two theorems, let $R=S\left[T_{i, j} \mid 1 \leq i \leq s, 1 \leq j \leq r_{i}\right]$. Then $R$ is equipped with a $G \times \mathbb{Z}^{s}$-graded structure in which $\operatorname{deg}_{G \times \mathbb{Z}^{s}}\left(x_{i}\right)=\left(\operatorname{deg}_{G}\left(x_{i}\right), 0\right)$ and $\operatorname{deg}_{G \times \mathbb{Z}^{s}}\left(T_{i, j}\right)=\left(0, e_{i}\right)$, where $e_{i}$ is the $i$ th element in the canonical basis of $\mathbb{Z}^{s}$. Recall that $\gamma_{i}=\operatorname{deg}_{G}\left(f_{i, j}\right)$ and let $\gamma:=\left(\gamma_{1}, \ldots, \gamma_{s}\right)$. For $t=\left(t_{1}, \ldots, t_{s}\right) \in \mathbb{Z}^{s}$, let $I^{t}:=I_{1}^{t_{1}} \ldots I_{s}^{t_{s}}, T^{t}:=T_{1}^{t_{1}} \ldots T_{s}^{t_{s}}, I^{t} T^{t}(\gamma . t):=I_{1}^{t_{1}}\left(t_{1} \gamma_{1}\right) T_{1}^{t_{1}} \ldots I_{s}^{t_{s}}\left(t_{s} \gamma_{s}\right) T_{s}^{t_{s}}$ and $M I^{t} T^{t}(\gamma . t):=M I_{1}^{t_{1}}\left(t_{1} \gamma_{1}\right) T_{1}^{t_{1}} \ldots I_{s}^{t_{s}}\left(t_{s} \gamma_{s}\right) T_{s}^{t_{s}}$.

Theorem 3.3. Assume that $i=0$ or $A$ is Noetherian. Then there exists a finite set $\Delta_{i} \subseteq G$ such that

(1) For all $t \in \mathbb{N}^{s}$, $\operatorname{Tor}_{i}^{S}\left(M I_{1}^{t_{1}} \cdots I_{s}^{t_{s}}, A\right)_{\eta}=0$ if $\eta \notin \Delta_{i}+t_{1} \gamma_{1}+\cdots+t_{s} \gamma_{s}$.

(2) There exists a subset $\Delta_{i}^{\prime} \subseteq \Delta_{i}$ such that $\operatorname{Tor}_{i}^{S}\left(M I_{1}^{t_{1}} \cdots I_{s}^{t_{s}}, A\right)_{\eta+t_{1} \gamma_{1}+\cdots+t_{s} \gamma_{s}} \neq 0$ for $t \gg 0$ and $\eta \in \Delta_{i}^{\prime}$ and $\operatorname{Tor}_{i}^{S}\left(M I_{1}^{t_{1}} \cdots I_{s}^{t_{s}}, A\right)_{\eta+t_{1} \gamma_{1}+\cdots+t_{s} \gamma_{s}}=0$ for $t \gg 0$ and $\eta \notin \Delta_{i}^{\prime}$.

(3) If, furthermore, $A \rightarrow k$ is a ring homomorphism to a field $k$, then for any $\delta$ and any $j$, the function

$$
\operatorname{dim}_{k} \operatorname{Tor}_{j}^{A}\left(\operatorname{Tor}_{i}^{S}\left(M I_{1}^{t_{1}} \cdots I_{s}^{t_{s}}, A\right)_{\delta+t_{1} \gamma_{1}+\cdots+t_{s} \gamma_{s}}, k\right)
$$

is polynomial in the $t_{i} s$ for $t \gg 0$, and the function

$$
\operatorname{dim}_{k} \operatorname{Tor}_{i}^{S}\left(M I_{1}^{t_{1}} \cdots I_{s}^{t_{s}}, k\right)_{\delta+t_{1} \gamma_{1}+\cdots+t_{s} \gamma_{s}}
$$

is polynomial in the $t_{i} s$ for $t \gg 0$.

Proof. Let $\mathcal{R}:=\bigoplus_{t \geq 0} I^{t} T^{t}(\gamma . t)$ and $M \mathcal{R}:=\bigoplus_{t>0} M I^{t} T^{t}(\gamma \cdot t)$ denote the (shifted) multi-Rees algebra and the multi-Rees module with respect to $I_{1}, \ldots, I_{s}$, and $M$. The natural surjective map $\phi: R \rightarrow \mathcal{R}$ that sends $x_{i}$ to $x_{i}$ and $T_{i, j}$ to $f_{i, j} T_{i}$ makes $M \mathcal{R}$ a finitely generated $G \times \mathbb{Z}^{s}$-graded module over $R$.

Observe that, for any $\delta \in G$ and $t \in \mathbb{Z}^{s}, M \mathcal{R}_{(\delta, t)} \simeq\left[M I^{t}(\gamma . t)\right]_{\delta}=\left[M I^{t}\right]_{\delta+\gamma . t}$, where in the last term $\delta+\gamma \cdot t:=\delta+t_{1} \gamma_{1}+\cdots+t_{s} \gamma_{s}$. Thus, the assertion follows by applying Theorem 3.1 to the $R$-module $\mathbb{M}:=M \mathcal{R}$.

Theorem 3.4. Let $\mathfrak{b}$ be a $G$-homogeneous ideal in $S$ such that for any $i \geq 0$ and $\delta \in G, H_{\mathfrak{b}}^{i}(S)_{\delta}$ is a finitely generated $A$-module. Then, if $A$ is Noetherian, for $i \geq 0$, there exists a subset $\Lambda_{i} \subseteq G$ such that

(1) $H_{\mathfrak{b}}^{i}\left(M I_{1}^{t_{1}} \cdots I_{s}^{t_{s}}\right)_{\eta+t_{1} \gamma_{1}+\cdots+t_{s} \gamma_{s}} \neq 0$ for $t=\left(t_{1}, \ldots, t_{s}\right) \gg 0$ if only if $\eta \in \Lambda_{i}$.

(2) If, furthermore, $A \rightarrow k$ is a ring homomorphism to a field $k$, then for any $\delta$ and any $j$, the function

$$
\operatorname{dim}_{k} \operatorname{Tor}_{j}^{A}\left(H_{\mathfrak{b}}^{i}\left(M I_{1}^{t_{1}} \cdots I_{s}^{t_{s}}\right)_{\delta+t_{1} \gamma_{1}+\cdots+t_{s} \gamma_{s}}, k\right)
$$

is a polynomial in the $t_{i} s$ for $t \gg 0$.

Proof. Since taking local cohomology respects the $G$-homogeneous degree, we have

$$
H_{\mathfrak{b}}^{i}\left(M I^{t}\right)_{\delta+\gamma \cdot t}=H_{\mathfrak{b} R}^{i}(M \mathcal{R})_{(\delta, t)}=\left(H_{\mathfrak{b} R}^{i}(M \mathcal{R})_{(\delta, *)}\right)_{t} .
$$


Let $B=A\left[T_{i, j}, 1 \leq i \leq s, 1 \leq j \leq r_{i}\right]$. Since $B$ is a flat extension of $A$, $H_{\mathfrak{b} R}^{i}(R)_{(\delta, *)}=H_{\mathfrak{b} R}^{i}\left(B \otimes_{A} S\right)_{(\delta, *)}$ is a finitely generated $B$-module. Let $\mathbb{F} \bullet$ be the minimal $G \times \mathbb{Z}^{s}$-graded free resolution of $M \mathcal{R}$ over $R$. Since $A$ is Noetherian, each term $\mathbb{F}_{j}$ of $\mathbb{F}_{\bullet}$ is of finite rank. This implies that for all $\delta \in G, i \geq 0$ and $j \geq 0, H_{\mathfrak{b} R}^{i}\left(\mathbb{F}_{j}\right)_{(\delta, *)}$ is a finitely generated $B$-module. The spectral sequence $H_{\mathfrak{b} R}^{i}\left(\mathbb{F}_{j}\right) \Rightarrow H_{\mathfrak{b} R}^{i-j}(M \mathcal{R})$ implies that $H_{\mathfrak{b} R}^{i}(M \mathcal{R})_{(\delta, *)}$ is a finitely generated multigraded $B$-module. This proves $(2)$ in view of $[11$, Theorem 1$]$.

Note that $H_{\mathfrak{b} R}^{i}(M \mathcal{R})_{(\delta, t)}=0$ for all $t \gg 0$ if and only if $K_{\delta}=H_{\mathfrak{b} R}^{i}(M \mathcal{R})_{(\delta, *)}$ is annihilated by a power of the ideal $\mathfrak{a}:=\bigcap_{i=1}^{s}\left(T_{i, 1}, \ldots, T_{i, r_{i}}\right)$. Hence (1) holds with

$$
\Lambda_{i}:=\left\{\delta \in G \mid K_{\delta} \neq H_{\mathfrak{a}}^{0}\left(K_{\delta}\right)\right\} .
$$

\section{Forms of arbitrary degrees}

This section is devoted to proving our main result in its full generality, when the ideals $I_{i}$ s are generated in arbitrary degrees. We start by recalling the notion of a Stanley decomposition of multigraded modules.

Definition 4.1. Let $\mathbb{G}$ be a finitely generated abelian group and let $B=A\left[T_{1}, \ldots, T_{r}\right]$ be a $\mathbb{G}$-graded polynomial ring over a commutative ring $A$. Let $M$ be a finitely generated $\mathbb{G}$-graded $B$-module. A Stanley decomposition of $M$ is a finite decomposition of the form

$$
M=\bigoplus_{i=1}^{m} u_{i} A\left[Z_{i}\right]
$$

where the direct sum is as $A$-modules, $u_{i}$ s are $\mathbb{G}$-homogeneous elements in $M, Z_{i} \mathrm{~s}$ are subsets (could be empty) of the variables $\left\{T_{1}, \ldots, T_{r}\right\}$, and $u_{i} A\left[Z_{i}\right]$ denotes the $A$-submodule of $M$ generated by elements of the form $u_{i} m$ where $m$ is a monomial in the polynomial ring $A\left[Z_{i}\right]$.

The following lemma is well known in $\mathbb{N}$-graded or standard $\mathbb{N}^{n}$-graded situations (cf. $[2,3,10]$ ).

Lemma 4.2. Let $\mathbb{G}$ be a finitely generated abelian group and let $B=A\left[T_{1}, \ldots, T_{r}\right]$ be a $\mathbb{G}$-graded polynomial ring. Let $I$ be a monomial ideal in $B$. Then a Stanley decomposition of $B / I$ exists.

Proof. The proof follows along the same lines as in the proof of [10, Corollary 6.4] or [2, Theorem 2.1], as one notices that any monomial is a homogeneous element.

Theorem 4.3. Let $\mathbb{G}$ be a finitely generated abelian group, $B=A\left[T_{1}, \ldots, T_{r}\right]$ be a $\mathbb{G}$-graded polynomial ring over a commutative ring $A$ and $\mathbb{M}$ be a finitely generated $\mathbb{G}$ graded B-module. Let $\Gamma$ denote the set of subsets of $\left\{\operatorname{deg}_{\mathbb{G}}\left(T_{i}\right)\right\}_{i=1}^{r}$ whose elements are linearly independent over $\mathbb{Z}$. Then there exists a collection of pairs $\left(\delta_{p}, E_{p}\right) \in \mathbb{G} \times \Gamma$, for $p=1, \ldots, m$, such that

$$
\operatorname{Supp}_{\mathbb{G}}(\mathbb{M})=\bigcup_{p=1}^{m}\left(\delta_{p}+\left\langle E_{p}\right\rangle\right),
$$

where $\left\langle E_{p}\right\rangle$ represents the free submonoid of $\mathbb{G}$ generated by elements in $E_{p}$. 
Proof. Since $\mathbb{M}$ is a finitely generated $\mathbb{G}$-graded $B$-module, there exists a homogeneous surjective map $\phi: F \rightarrow \mathbb{M}$ from a free $B$-module $F$ to $\mathbb{M}$. We can write $F=\bigoplus_{i=1}^{m} B e_{i}$, where $\operatorname{deg}_{G}\left(e_{i}\right)$ represents the degree of the $i$ th generator of $M$. Let $K=\operatorname{ker} \phi$. Then $\mathbb{M} \simeq F / K$. In particular, $\operatorname{Supp}_{\mathbb{G}}(\mathbb{M})=\operatorname{Supp}_{\mathbb{G}}(F / K)$.

Extend any monomial order on $B$ to a monomial order on $F=\bigoplus_{i=1}^{m} B e_{i}$, by ordering the $e_{i}$ 's. Since $\mathbb{M}$ is $\mathbb{G}$-graded, so is $K$. Thus, by Proposition 2.7, we have $\operatorname{Supp}_{\mathbb{G}}(F / K)=\operatorname{Supp}_{\mathbb{G}}\left(F / \operatorname{in}_{\prec}(K)\right)$. Therefore,

$$
\operatorname{Supp}_{\mathbb{G}}(\mathbb{M})=\operatorname{Supp}_{\mathbb{G}}\left(F / \operatorname{in}_{\prec}(K)\right) .
$$

Observe that $\operatorname{in}_{\prec}(K)$ is generated by monomials of the form $\mathbf{T}^{\alpha} e_{i}$ (where $\mathbf{T}^{\alpha}=$ $T_{1}^{\alpha_{1}} \cdots T_{r}^{\alpha_{r}}$ for $\left.\alpha=\left(\alpha_{1}, \ldots, \alpha_{r}\right) \in \mathbb{N}^{r}\right)$. Let $I_{i}$ be the monomial ideal in $B$ generated by all monomials $\mathbf{T}^{\alpha}$ for which $\mathbf{T}^{\alpha} e_{i} \in \operatorname{in}_{\prec}(K)$. Clearly, $F / \operatorname{in}_{\prec}(K) \simeq \bigoplus_{i=1}^{m} \frac{B}{I_{i}} e_{i}$.

By Lemma 4.2, for each $i=1, \ldots, m$, there exists a Stanley decomposition of $\frac{B}{I_{i}} e_{i}$

$$
\frac{B}{I_{i}} e_{i} \simeq \bigoplus_{j=1}^{m_{i}} u_{i j} A\left[T_{i j}\right]
$$

where $u_{i j}$ are homogeneous elements of $\frac{B}{I_{i}} e_{i}$ and $T_{i j}$ are subsets (could be empty) of the variables $\left\{T_{1}, \ldots, T_{r}\right\}$ in $B$. This gives

$$
F / \operatorname{in}_{\prec}(K) \simeq \bigoplus_{i=1}^{m} \bigoplus_{j=1}^{m_{i}} u_{i j} A\left[T_{i j}\right] .
$$

Thus, $\operatorname{Supp}_{\mathbb{G}}\left(F / \operatorname{in}_{\prec}(K)\right)$ can be written as a finite union of the form

$$
\operatorname{Supp}_{\mathbb{G}}\left(F / \operatorname{in}_{\prec}(K)\right)=\bigcup_{i, j} \operatorname{Supp}_{\mathbb{G}}\left(u_{i j} A\left[T_{i j}\right]\right) .
$$

Let $\delta_{i j}=\operatorname{deg}_{\mathbb{G}}\left(u_{i j}\right)$. Then

$$
\operatorname{Supp}_{\mathbb{G}}\left(F / \operatorname{in}_{\prec}(K)\right)=\bigcup_{i, j}\left(\delta_{i j}+\operatorname{Supp}_{\mathbb{G}}\left(A\left[T_{i j}\right]\right)\right) .
$$

To prove the theorem, it now suffices to show that $\operatorname{Supp}_{\mathbb{G}}\left(A\left[T_{i j}\right]\right)$ can be decomposed into a union of free submonoids of $\mathbb{G}$ of the form $\langle E\rangle$, where $E$ is a linearly independent subset in $\Gamma$.

Since $A\left[T_{i j}\right]$ is a polynomial ring whose variables are variables of $B$, without loss of generality, we may assume that $T_{i j}=\left\{T_{1}, \ldots, T_{r}\right\}$, i.e., $A\left[T_{i j}\right]=B$. Let $H$ be the binomial ideal in $B$ generated by $\left\{\mathbf{T}^{\alpha}-\mathbf{T}^{\beta} \mid \operatorname{deg}_{\mathbb{G}}\left(\mathbf{T}^{\alpha}\right)=\operatorname{deg}_{\mathbb{G}}\left(\mathbf{T}^{\beta}\right)\right\}$. Then taking the quotient $B / H$ is the same as identifying monomials of the same degree in $B$. Thus, we have

$$
\operatorname{Supp}_{\mathbb{G}}(B)=\operatorname{Supp}_{\mathbb{G}}(B / H)=\operatorname{Supp}_{\mathbb{G}}\left(B / \operatorname{in}_{\prec}(H)\right)
$$

and

$$
\left(\frac{B}{H}\right)_{\gamma}=\left(\frac{B}{\operatorname{in}_{\prec}(H)}\right)_{\gamma}= \begin{cases}A & \text { if } \gamma \in \operatorname{Supp}_{\mathbb{G}}(B / H), \\ 0 & \text { otherwise. }\end{cases}
$$


By Lemma 4.2, a Stanley decomposition of $B /$ in $_{\prec}(H)$ exists. That is, we can write

$$
B / \operatorname{in}_{\prec}(H)=\bigoplus_{j=1}^{s} u_{j} A\left[Z_{j}\right]
$$

where $u_{j}$ are $\mathbb{G}$-homogeneous elements of $B /$ in $_{\prec}(H)$ and $Z_{j}$ are subsets (could be empty) of the variables $\left\{T_{1}, \ldots, T_{r}\right\}$. Let $E_{j}$ be the set of degrees of variables in $Z_{j}$. It further follows from (4.1) that $B /$ in $_{\prec}(H)$ has at most one monomial in each degree. This implies that the support of $u_{j} A\left[Z_{j}\right]$ are all disjoint and each set $E_{j}$ is linearly independent. Hence, by letting $\sigma_{j}=\operatorname{deg}_{\mathbb{G}}\left(u_{j}\right)$, we have

$$
\operatorname{Supp}_{\mathbb{G}}(B)=\coprod_{j=1}^{s}\left(\sigma_{j}+\left\langle E_{j}\right\rangle\right)
$$

The theorem is proved.

Remark 4.4. It would be nice if the union in Theorem 4.3 is a disjoint union. However, this is not true. Let $B=A[x, y]$ be a $\mathbb{Z}$-graded polynomial ring with $\operatorname{deg}(x)=4$ and $\operatorname{deg}(y)=7$ (hence, $\Gamma=\{4,7\})$. Let $\mathbb{M}=B /(x) \oplus B /(y) \simeq A[y] \oplus A[x]$. Then $\operatorname{Supp}_{\mathbb{Z}}(\mathbb{M})=\left\{4 a+7 b \mid a, b \in \mathbb{Z}_{\geq 0}\right\}$. Moreover, linearly independent subsets of $\Gamma$ are $\{4\}$ and $\{7\}$. It can be easily seen that $\operatorname{Supp}_{\mathbb{Z}}(\mathbb{M})$ cannot be written as disjoint union of shifted free submonoids of $\mathbb{Z}$ generated by 4 and/or by 7 .

For a vector $\mathbf{c}=\left(c_{1}, \ldots, c_{s}\right) \in \mathbb{Z}^{s}$ and a tuple $E=\left(\nu_{1}, \ldots, \nu_{s}\right)$ of elements in $G$, we shall denote $\Delta E$ the empty tuple if $s \leq 1$ and the $(s-1)$-tuple $\left(\nu_{2}-\nu_{1}, \ldots, \nu_{s}-\nu_{s-1}\right)$ else, and by c. $E$ the $G$-degree $\sum_{j=1}^{s} c_{j} \nu_{j}$. If $E$ and $E^{\prime}$ are tuples, we denote by $E \mid E^{\prime}$ the concatenation of $E$ and $E^{\prime}$.

Remark 4.5. With some simple linear algebra arguments, it can be seen that for tuples $E_{1}, \ldots, E_{s}$ of elements of $G$, the tuple of elements of $G \times \mathbb{Z}^{s}, E_{1} \times\left\{e_{1}\right\}|\cdots| E_{s} \times$ $\left\{e_{s}\right\}$, where $e_{i}$ is the $i$ th basis element of $\mathbb{Z}^{s}$, is linearly independent if and only if $\Delta E_{1}|\cdots| \Delta E_{s}$ is linearly independent. These equivalent conditions imply that for $\left(\mathbf{c}_{1}, \ldots, \mathbf{c}_{s}\right) \neq\left(\mathbf{c}_{1}^{\prime}, \ldots, \mathbf{c}_{s}^{\prime}\right)$, with $\mathbf{c}_{i}, \mathbf{c}_{i}^{\prime} \in \mathbb{Z}_{\geq 0}^{\left|E_{i}\right|}$ and $\left|\mathbf{c}_{i}\right|=\left|\mathbf{c}_{i}^{\prime}\right|$ for all $i$, one has $\mathbf{c}_{1} . E_{1}+$ $\cdots+\mathbf{c}_{s} \cdot E_{s} \neq \mathbf{c}_{1}^{\prime} \cdot E_{1}+\cdots+\mathbf{c}_{s}^{\prime} \cdot E_{s}$. This last fact is a direct corollary of the linear independence of $E_{1} \times\left\{e_{1}\right\}|\cdots| E_{s} \times\left\{e_{s}\right\}$, as $\mathbf{c}_{1} \cdot\left(E_{1} \times\left\{e_{1}\right\}\right)+\cdots+\mathbf{c}_{s} \cdot\left(E_{s} \times\left\{e_{s}\right\}\right)=$ $\left(\mathbf{c}_{1} \cdot E_{1}+\cdots+\mathbf{c}_{s} \cdot E_{s}\right) \times\left(\left|\mathbf{c}_{1}\right| e_{1}+\cdots+\left|\mathbf{c}_{s}\right| e_{s}\right)$.

We shall now prove our main result. Recall that $I_{i}=\left(f_{i, 1}, \ldots, f_{i, r_{i}}\right)$ and let $\gamma_{i, j}=$ $\operatorname{deg}_{G}\left(f_{i, j}\right)$.

Theorem 4.6. Let $G$ be a finitely generated abelian group and let $S=A\left[x_{1}, \ldots, x_{n}\right]$ be a G-graded algebra over a commutative ring $A \subseteq S_{0}$. Let $I_{i}=\left(f_{i, 1}, \ldots, f_{i, r_{i}}\right)$ for $i=1, \ldots s$ be $G$-homogeneous ideals in $S$, and let $M$ be a finitely generated $G$ graded $S$-module. Set $\Gamma_{i}=\left\{\operatorname{deg}_{G}\left(f_{i, j}\right)\right\}_{j=1}^{r_{i}}$. Let $\ell \geq 0$ and assume that $\ell=0$ or $A$ is Noetherian.

There exist a finite collection of elements $\delta_{p}^{\ell} \in G$, a finite collection of integers $t_{p, i}^{\ell}$, and a finite collection of non-empty tuples $E_{p, i}^{\ell} \subseteq \Gamma_{i}$, such that $\Delta E_{p, 1}^{\ell}|\cdots| \Delta E_{p, s}^{\ell}$ is 
linearly independent for all p, satisfying :

$$
\operatorname{Supp}_{G}\left(\operatorname{Tor}_{\ell}^{S}\left(M I_{1}^{t_{1}} \cdots I_{s}^{t_{s}}, A\right)\right)=\bigcup_{p=1}^{m}\left(\delta_{p}^{\ell}+\underset{\substack{\mathbf{c}_{i} \in \mathbb{Z}_{\geq 0}^{\left|E_{p, i}^{\ell},\right| \mathbf{c}_{i} \mid=t_{i}-t_{p, i}^{\ell}}}}{\bigcup} \mathbf{c}_{1} \cdot E_{p, 1}^{\ell}+\cdots+\mathbf{c}_{s} \cdot E_{p, s}^{\ell}\right),
$$

if $t_{i} \geq \max _{p}\left\{t_{p, i}^{\ell}\right\}$ for all $i$.

Proof. As before, we use $t$ to denote $\left(t_{1}, \ldots, t_{s}\right) \in \mathbb{Z}^{s}$, and let $\mathcal{R}:=\bigoplus_{t \geq 0} I^{t} T^{t}$ and $M \mathcal{R}:=\bigoplus_{t \geq 0} M I^{t} T^{t}$. Consider $R=A\left[x_{1}, \ldots, x_{n}\right]\left[T_{i, j}, 1 \leq i \leq s, 1 \leq j \leq r_{i}\right]$, the $G \times \mathbb{Z}^{s}$-graded polynomial ring over $A\left[x_{1}, \ldots, x_{n}\right]$ with $\operatorname{deg}_{G \times \mathbb{Z}^{s}}\left(x_{i}\right)=\left(\operatorname{deg}_{G}\left(x_{i}\right), 0\right)$ and $\operatorname{deg}_{G \times \mathbb{Z}^{s}}\left(T_{i, j}\right)=\left(\operatorname{deg}_{G}\left(f_{i, j}\right), e_{i}\right)$, where $e_{i}$ denotes the $i$ th canonical generator of $\mathbb{Z}^{s}$. The natural surjective map $\phi: R \rightarrow \mathcal{R}$ that sends $x_{i}$ to $x_{i}$ and $T_{i, j}$ to $f_{i, j} T_{i}$ makes $M \mathcal{R}$ a finitely generated $G \times \mathbb{Z}^{s}$-graded module over $R$.

Let $\mathbb{F}$. be a $G \times \mathbb{Z}^{s}$-graded free resolution of $M \mathcal{R}$ over $R$. If $A$ is Noetherian, then each $\mathbb{F}_{i}$ can be chosen of finite rank, and we make such a choice. For $t \in \mathbb{Z}^{s}$, the degree $(*, t)$-strand $\mathbb{F}_{\bullet}^{t}$ of $\mathbb{F}_{\bullet}$. provides a $G$-graded free resolution of $M I^{t}$ over $S=R_{(*, 0)}$. Thus,

$$
\operatorname{Tor}_{i}^{S}\left(M I^{t}, A\right)=H_{i}\left(\mathbb{F}_{\bullet}^{t} \otimes_{S} A\right)
$$

Moreover, taking homology respects the graded structure, and therefore,

$$
H_{i}\left(\mathbb{F}^{t} \bullet \otimes_{S} A\right)=H_{i}\left(\mathbb{F} \bullet \otimes_{R} R / \mathfrak{m} R\right)_{(*, t)},
$$

where $\mathfrak{m}=\left(x_{1}, \ldots, x_{n}\right)$ is the homogeneous irrelevant ideal in $S$.

Let $\Gamma^{\prime}=\left\{\left(\gamma_{i, j}, e_{i}\right) \in G \times \mathbb{Z}^{s}\right\}=\coprod_{i} \Gamma_{i} \times\left\{e_{i}\right\}$ be the set of degrees of the variables $T_{i, j}$. Observe that $H_{i}\left(\mathbb{F} \bullet \otimes_{R} R / \mathfrak{m} R\right)$ is a finitely generated $G \times \mathbb{Z}^{s}$-graded module over $R / \mathfrak{m} R \simeq B$ for any $i$ if $A$ is Noetherian, and for $i=0$ in any case. Applying Theorem 4.3 to the $G \times \mathbb{Z}^{s}$-graded module $H_{i}\left(\mathbb{F} \bullet \otimes_{R} R / \mathfrak{m} R\right)$ we obtain a finite collection of elements $\theta_{p}^{\ell} \in G \times \mathbb{Z}^{s}$ and a finite collection of linearly independent subsets $E_{p}^{\ell} \subseteq \Gamma^{\prime}$ (which we view in a fixed order as tuples), for $p=1, \ldots, m$, such that

$$
\operatorname{Supp}_{G \times \mathbb{Z}^{s}}\left(H_{i}\left(\mathbb{F} \bullet \otimes_{R} R / \mathfrak{m} R\right)\right)=\bigcup_{p=1}^{m}\left(\theta_{p}^{\ell}+\left\langle E_{p}^{\ell}\right\rangle\right)
$$

Let $\theta_{p}^{\ell}=\left(\delta_{p}^{\ell}, t_{1, p}^{\ell}, \ldots, t_{s, p}^{\ell}\right)$, where $\delta_{p}^{\ell} \in G$ and $t_{i, p}^{\ell} \in \mathbb{Z}$. One has $E_{p}^{\ell}=\coprod_{i=1}^{s} E_{p, i}^{\ell} \times\left\{e_{i}\right\}$. The linear independence of the elements in $E_{p}^{\ell}$ is equivalent to the fact that the elements of $\Delta E_{p, 1}^{\ell}|\cdots| \Delta E_{p, s}^{\ell}$ are linearly independent.

Taking the degree $(*, t)$-strand of $H_{i}\left(\mathbb{F} \bullet \otimes_{R} R / \mathfrak{m} R\right)$, we get

$$
\operatorname{Supp}_{G}\left(\operatorname{Tor}_{\ell}^{S}\left(M I_{1}^{t_{1}} \cdots I_{s}^{t_{s}}, A\right)\right)=\bigcup_{p=1}^{m}\left(\delta_{p}^{\ell}+\underset{\substack{\mathbf{c}_{i} \in \mathbb{Z}_{\geq 0}^{\left|E_{p, i}^{\ell},\right| \mathbf{c}_{i} \mid=t_{i}-t_{p, i}^{\ell}}}}{\bigcup} \mathbf{c}_{1} \cdot E_{p, 1}^{\ell}+\cdots+\mathbf{c}_{s} \cdot E_{p, s}^{\ell}\right) .
$$


Example 4.7. Let $I \subseteq S$ be a complete intersection ideal of three forms $f_{1}, f_{2}, f_{3}$ of degrees $a, b, c$. One easily sees, for instance by the Hilbert Burch theorem, that

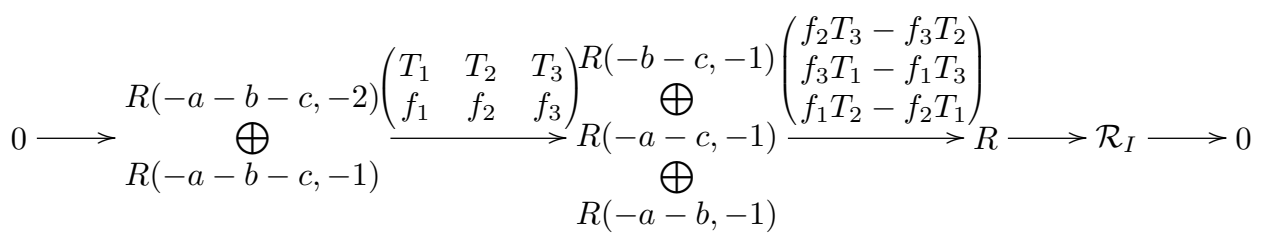

is a graded free $R$-resolution of $\mathcal{R}_{I}$.

It follows that $\operatorname{Tor}_{0}^{S}\left(I^{t}, A\right)_{\mu}=B_{\mu, t}, \operatorname{Tor}_{2}^{S}\left(I^{t}, A\right)_{\mu}=B_{\mu-a-b-c, t-1}$, and

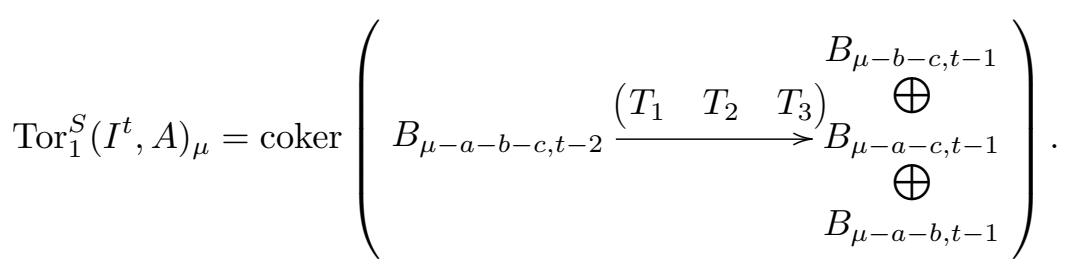

Now, $\operatorname{Tor}_{1}^{S}\left(\mathcal{R}_{I}, A\right)$ has the following Stanley decomposition:

$$
A\left[T_{1}, T_{2}, T_{3}\right](-b-c,-1) \bigoplus A\left[T_{1}, T_{3}\right](-a-c,-1) \bigoplus A\left[T_{1}, T_{2}, T_{3}\right](-a-b,-1) .
$$

The ideal $H$ generated by the binomials $T^{\alpha}-T^{\beta}$ with $\operatorname{deg}\left(T^{\alpha}\right)=\operatorname{deg}\left(T^{\beta}\right)$ is the kernel of the map

$$
\begin{gathered}
A\left[T_{1}, T_{2}, T_{3}\right] \longrightarrow A[u, v] \\
T_{1} \longrightarrow u v^{a} \\
T_{2} \longrightarrow u v^{b} \\
T_{3} \longrightarrow u v^{c}
\end{gathered}
$$

and is therefore generated by a single irreducible and homogeneous binomial.

If, for example, $(a, b, c)=(2,5,8)$ then this relation is $T_{2}^{2}-T_{1} T_{3}$, and a Stanley decomposition of, for instance, $A\left[T_{1}, T_{2}, T_{3}\right] /\left(T_{2}^{2}\right)$ is $A\left[T_{1}, T_{3}\right] \oplus T_{2} A\left[T_{1}, T_{3}\right](-5,-1)$. It finally gives the following decomposition for $\operatorname{Tor}_{1}^{S}\left(\mathcal{R}_{I}, A\right): A\left[T_{1}, T_{3}\right](-13,-1) \oplus$ $T_{2} A\left[T_{1}, T_{3}\right](-18,-2) \oplus A\left[T_{1}, T_{3}\right](-10,-1) \oplus A\left[T_{1}, T_{3}\right](-7,-1) \oplus T_{2} A\left[T_{1}, T_{3}\right](-12,-2)$.

Setting $E_{t}:=\left\{2 \alpha+8 \beta \mid \alpha, \beta \in \mathbb{Z}_{+}, \alpha+\beta=t\right\}=2 t+6\{0, \cdots, t\}$, one gets that for $t \geq 2$,

$$
\begin{aligned}
- & \operatorname{Supp}_{\mathbb{Z}}\left(\operatorname{Tor}_{0}^{S}\left(I^{t}, A\right)\right)=E_{t} \cup\left(5+E_{t-1}\right), \\
- & \operatorname{Supp}_{\mathbb{Z}}\left(\operatorname{Tor}_{1}^{S}\left(I^{t}, A\right)\right)=\left(13+E_{t-1}\right) \cup\left(18+E_{t-2}\right) \cup\left(10+E_{t-1}\right) \cup\left(7+E_{t-1}\right) \cup \\
& \quad\left(12+E_{t-2}\right), \\
- & \operatorname{Supp}_{\mathbb{Z}}\left(\operatorname{Tor}_{2}^{S}\left(I^{t}, A\right)\right)=\left(15+E_{t-1}\right) \cup\left(20+E_{t-2}\right) .
\end{aligned}
$$$$
\text { Note that one has the simplified expression : }
$$$$
-\operatorname{Supp}_{\mathbb{Z}}\left(\operatorname{Tor}_{1}^{S}\left(I^{t}, A\right)\right)=\left(5+E_{t}\right) \cup\left(10+E_{t-1}\right) \text {. }
$$

\section{Acknowledgments}

Our work was done when H. Tài Hà visited the other authors at the Institut de Mathématiques de Jussieu, UPMC. The authors would like to thank the Institut for its support and hospitality. H. Tài Hà is partially supported by NSA grant H9823011-1-0165. 


\section{References}

[1] D. Berlekamp, Regularity defect stabilization of powers of an ideal, 2011, arXiv:1105.2260.

[2] K. Baclawski and A.M. Garsia, Combinatorial decompositions of a class of rings, Adv. Math. 39 (1981), 155-184.

[3] W. Bruns, C. Krattenthaler and J. Uliczka, Stanley decompositions and Hilbert depth in the Koszul complex, J. Comm. Algebra 2(3) (2010), 327-357.

[4] M. Chardin, Powers of ideals and the cohomology of stalks and fibers of morphisms, 2010, arXiv: 1009.1271.

[5] S.D. Cutkosky, J. Herzog and N.V. Trung, Asymptotic behaviour of the Castelnuovo-Mumford regularity. Compost. Math. 118 (1999), 243-261.

[6] D. Eisenbud, Commutative Algebra: with a View Toward Algebraic Geometry, Springer-Verlag, New York, 1995.

[7] D. Eisenbud and J. Harris, Powers of ideals and fibers of morphisms, Math. Res. Lett. 17(2) (2010), 267-273.

[8] D. Eisenbud and B. Ulrich, Stabilization of the regularity of powers of an ideal, 2010, arXiv: 1012.0951.

[9] H.T. Hà, Asymptotic linearity of regularity and $a^{*}$-invariant of powers of ideals, Math. Res. Lett. 18(1) (2011), 1-9.

[10] J. Herzog and D. Popescu, Finite filtrations of modules and shellable multicomplexes, Manuscripta Math. 121(3) (2006), 385-410.

[11] V. Kodiyalam, Homological invariants of powers of an ideal. Proc. Amer. Math. Soc. 118(3) (1993), 757-764.

[12] V. Kodiyalam, Asymptotic behaviour of Castelnuovo-Mumford regularity, Proc. Amer. Math. Soc. 128(2) (1999), 407-411.

[13] H. Matsumura, Commutative Ring Theory, Cambridge Stud. in adv. math. 8, Cambridge University Press, Cambridge, 1986.

[14] P. Singla, Convex-geometric, Homological and Combinatorial Properties of Graded Ideals, Genehmitge Dissertation, Universität Duisburg-Essen, December, 2007.

[15] N.V. Trung and H. Wang, On the asymptotic behavior of Castelnuovo-Mumford regularity. J. Pure Appl. Algebra 201(1-3) (2005), 42-48.

[16] G. Whieldon, Stabilization of Betti Tables, 2011, arXiv:1106.2355.

Institut de Mathématiques de Jussieu, UPMC, Boite 247, 4 Place Jussieu, F-75252 Paris Cedex, France

E-mail address: bagheri@math.jussieu.fr

E-mail address: chardin@math.jussieu.fr, http://people.math.jussieu.fr/ chardin

Department of Mathematics, Tulane University, 6823 St. Charles Avenue, New Orleans, LA 70118, USA

E-mail address: tha@tulane.edu, www.math.tulane.edu/ tai/ 